\title{
Multi-Head Track-Sector Clustering Routing Algorithm In WSN
}

\author{
Qiaozhi $\mathrm{Xu}^{1, \mathrm{a}^{*}}$, Jie Zhao ${ }^{2, \mathrm{~b}}$ \\ ${ }^{1}$ College of Computer Science, Inner Mongolia University, Hohhot, China \\ ${ }^{2}$ College of Math \& Information Technology, Jiangsu Second Normal University, Nanjing, China \\ a ciecxqz@imnu.edu.cn, ${ }^{b} 55239353 @ q q . c o m$
}

Keywords: multi-head leach protocol; track-sector clustering algorithm; wsn routing algorithm Abstract. Routing is one of the critical technologies in WSNs, and many routing protocols have been proposed in order to achieve the energy efficiency in WSN. Based on CCS, TSC scheme divided the cluster smaller, shorten the chain length and saved more energy than CCS. But the chain is still long which resulting in the larger delay. We propose MH-TSC scheme to improve the TSC routing algorithm, mainly including: (1) selecting multi heads for a cluster to balance the energy consumption; (2) these heads construct a shorter chain to reduce the delay. The analysis and performance evaluation prove that the MH-TSC can further reduce the energy depletion in the network and prolong the network life.

\section{Introduction}

With the development of the Micro-Electro-Mechanical System (MEMS) and wireless communication technology, wireless sensor networks (WSNs) are becoming increasingly attractive for numerous application areas, and WSNs have been managed to establish the connection between the physical world, the computing world and human society. In general, a WSN consists of many tiny sensor nodes distributed over a large area with one or more powerful sinks or base stations (BSs) collecting information from these sensor nodes. All sensor nodes have limited power supply and can do information sensing, data processing and wireless communication.

Routing is one of the critical technologies in WSNs, and many routing protocols have been proposed in order to achieve the energy efficiency in WSN. Based on network structure, routing protocols in WSNs can be coarsely divided into two categories: flat routing and hierarchical routing. Flat routing protocols are relatively effective in small scale networks. In hierarchical routing protocols, the network is divided into several logical groups called a cluster. Sensor nodes collect the information in the cluster and the head node aggregates the information to decrease the amount of the data. Each sensor node delivers the sensing data to one or more head nodes in the cluster and the head node delivers the aggregated data to the BS [1]. Hierarchical routing protocols have significant savings in total energy consumption of the WSN.

Popular routing protocols based on clustering schemes, such as LEACH, energy LEACH, multi-hop LEACH, LEACH-C, PEGASIS, CCS and TSC scheme proposed in [2-7] are effective in conserving energy. However, these routing protocols have energy consumption loopholes and unequal depletion of energy in head nodes.

We propose MH-TSC scheme to improve the TSC routing algorithm, by setting multi heads in a cluster, further reducing the energy depletion in the network. Also, the proposed MH-TSC contributes to the conservation of energy by reducing the number of data messages aggregation at the head node.

The rest of the paper is organized as follows: firstly, we describe the related routing protocols motivated the MH-TSC scheme, then we present the proposed MH-TSC scheme, following we compare the energy consumption of the MH-TSC scheme and TSC, finally, we conclude the MH-TSC scheme. 


\section{Related Work}

LEACH [2] is one of the pioneering clustering routing approaches for WSNs, its operation is broken up into lots of rounds, and each round is divided into two phases, the set-up phase and the steady-state phase. In the set-up phase the clusters are organized, while in the steady-state phase data is delivered to the BS. LEACH uses randomized rotation method for selection of head node to distribute energy consumption uniformly in the network, but the CHs performs single-hop to the BS, which is not applicable to large-region network, as the long-range communications directly from $\mathrm{CHs}$ to the $\mathrm{BS}$ will consume too much energy; on the other hand, $\mathrm{CHs}$ are elected in terms of probabilities without energy considerations, it is hard to ensure the $\mathrm{CHs}$ to be uniformly distributed throughout the network, and this could bring about energy holes and coverage problems.

PEGASIS [5] is an improvement of LEACH. The locations of nodes are random and are organized to form a chain and take turns being the $\mathrm{CH}$ for transmission to the BS. Each node receives data from its neighbor, fuses it with its own data, and transmits to its one-hop neighbor on the chain, this process is repeated until the $\mathrm{CH}$ gets data from all the nodes. The head node is selected with the node number obtained by calculating $\mathrm{i}$ modN , where $\mathrm{N}$ represents the number of nodes and $\mathrm{i}$ represents the current round number [5]. PEGASIS achieves energy conservation by reducing the number of data messages gathering at $\mathrm{CH}$ and distance of the data transmitted, but there are still some loopholes for energy consumption [6].

To reduce the energy consumption loopholes in PEGASIS, a protocol called CCS has been proposed in [6]. In CCS, the network is divided into a variety of concentric circular tracks which represent different clusters and each circular track is assigned with a level. The track nearest to the BS is assigned with level-1 and the level number increases with the increase of the distance to the BS. Chains are constructed within the track as in PEGASIS. One of the nodes on the chain at each level area is selected as a $\mathrm{CH}$. A CH in level $\mathrm{L}$ is selected with node number obtained by calculating $\mathrm{modML}$, where MLrepresents the number of nodes that have the same level in i round. All the nodes receive data from one-hop neighbor and fuse with their own data, and then transmit to the next neighbor in the chain. The $\mathrm{CH}$ then transmits the data to the $\mathrm{CH}$ in the lower level track, At last, level $1 \mathrm{CH}$ transmits these data to the BS. Fig.1 shows the basic topology of CCS scheme. Compared to PEGASIS, CCS saves a considerable amount of energy [7]. But Residual energy is not considered for $\mathrm{CH}$ election, which may lead to unbalanced energy consumption among all nodes; secondly, chains formed in the clusters are long over the concentric circles, therefore, the data has to flow around the BS over a long circular chain before reaching to the BS. Also, the distance between the CHs and the distance between the $\mathrm{CH}$ in level-1 track and the BS could still be larger in the case of large and non-uniform network, this could consume more energy in the network [7].

TSC [7] is an improvement of CCS. TSC scheme divides the network into concentric circular tracks and triangular sectors, uses tracks and sectors to form clusters, and thus breaks the large circular chain into smaller clusters. One $\mathrm{CH}$ is selected in each cluster. Chains are constructed within each cluster area formed by the intersection of tracks and sectors. Fig. 2 shows the basic topology of TSC scheme. Compared to CCS, TSC reduces the redundant data transmission by breaking the large circular chain into smaller clusters, also reduces the energy consumption caused by large distance between $\mathrm{CH}$ and the BS. But residual energy is still not considered for $\mathrm{CH}$ election, and Chain-based protocols, enable nodes to communicate with their nearest neighbor by using low radio power, but long chain would cause large delay [8].

Motivated by these scheme, we propose the MH-TSC scheme to improve the TSC scheme by selecting multi $\mathrm{CHs}$ for each cluster, which balances the energy consumption of the $\mathrm{CH}$ nodes and network load in the WSN which is not presented in the above schemes. 


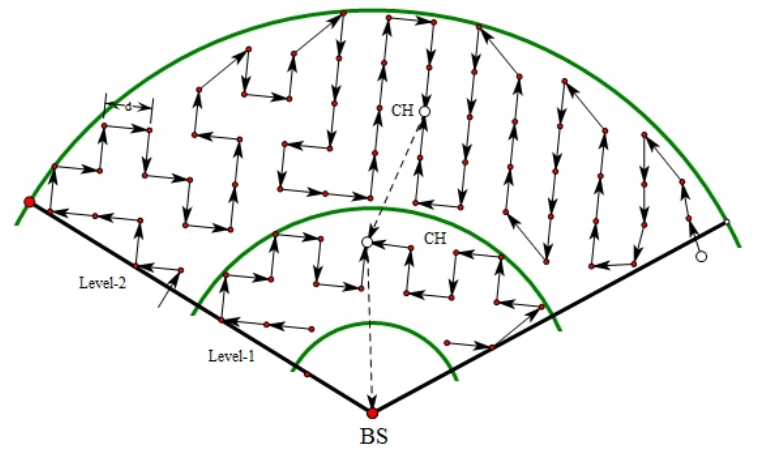

Fig 1. Data transmitting in CCS

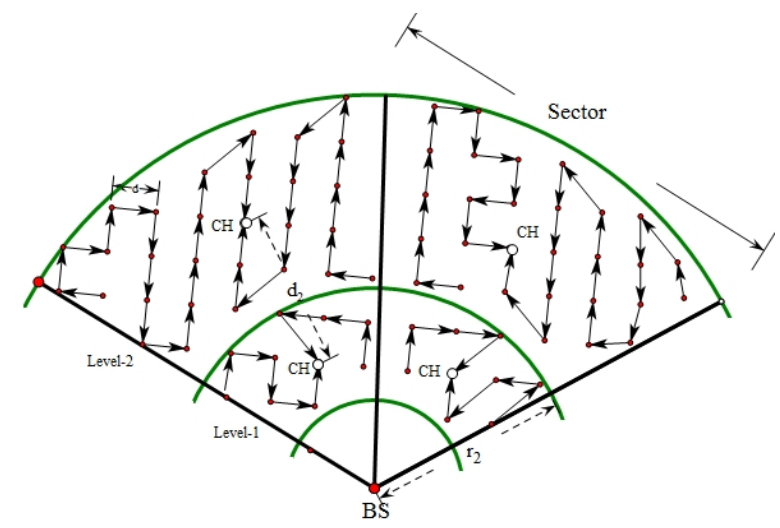

Fig 2. Data Transmitting in TSC

\section{MH-TSC Scheme}

The MH-TSC scheme also uses tracks and sectors to form clusters, therefore, a cluster is an area under curved strip formed by the intersection of a circular track and a triangular sector. In MH-TSC, there exists one major $\mathrm{CH}$ and several auxiliary $\mathrm{CH}$ in each cluster and they construct a chain. The aux-CHs collect and fuse the information from the nodes, and transmit to the major- $\mathrm{CH}$, then the major- $\mathrm{CH}$ transmits the data to the BS. Compared to one $\mathrm{CH}$ in a cluster of TSC, multi CHs share the workload together, balance the energy consumption and network load in a cluster, and the shorter chain avoid the large delay described in [8].

The MH-TSC operation is divided into three phases: cluster partition and head selection, Chain Construction and data transmission.

Network Model Assumptions:

1) The nodes distribute in an area randomly and evenly;

2) The BS is in the center of the network with unlimited energy, enough processing power, and the position is fixed;

3) All the sensor nodes are homogeneous and their location are fixed;

4) Every sensor node has a unique ID;

5) The energy of the sensor node is limited and can't be supplementary;

6) The sensor nodes can adjust their transmit power according to the transmission distance;

7) The sensor node has GPS functions.

A. Cluster Partition and Head Selection.The formation of track and sector depends on the position of the nodes in order to ensure the uniform cluster size.

Initially, all nodes report their position and residual energy to the BS, the BS divides the sensor network into some levels, and the level is given as the form like a concentric circle. The number of these levels is depended on various parameters such as the density of the sensor networks, the number of nodes, or the location of the BS [6].The track nearest to the BS is leveled as level-1 and the tracks with higher level are further away from the BS. The track setup remains unchanged throughout the network lifetime.

Secondly, the BS constructs the sectors over the network. The number of sectors depends upon various factors too, such as distribution density of the network, number of nodes, distance among the nodes, and the transmission delay [7]. In MH-TSC, the number of sectors is determined initially in BS. According to [7], we also select the number of sectors that the angle projected at the BS by each sector is $60^{\circ}$, thus, the distance between any two nodes in the sector is limited within the length equal to the radius of the highest level track. The sector setup remains unchanged throughout the network lifetime.

Now some clusters under curved strip formed by the intersection of a circular track and a triangular sector are divided, and each cluster has a cluster ID. The BS broadcasts the partition results to all nodes, so each node knows its cluster ID.

Following, the BS selects $\mathrm{N}$ nodes with largest residual energy as the $\mathrm{CHs}$ of the cluster, $\mathrm{N}$ is determined previously, and in our experiments, the $\mathrm{CH}$ number in a cluster is set 3 . 
The BS broadcasts the results to all the nodes, including cluster-ID, CHs ID and their position.

B. Chain Construction. When all the nodes receive the above information from the BS, according to the position, the $\mathrm{CHs}$ constructs a chain and the node in the middle becomes the major $\mathrm{CH}$, and the others are auxiliary $\mathrm{CH}$ (aux- $\mathrm{CH}$ ), the aux- $\mathrm{CHs}$ are responsible for collecting and fusing data from the member nodes and transferring to the one-hop neighbor in the chain, and the major $\mathrm{CH}$ aggregates data from two neighbored aux-CHs in the chain and major $\mathrm{CH}$ of upper level, then forwards to the major $\mathrm{CH}$ in lower level.

Fig. 3 shows the basic topology of MH-TSC scheme. The chain is shorter than TSC and CCS, so the delay is smaller than them.

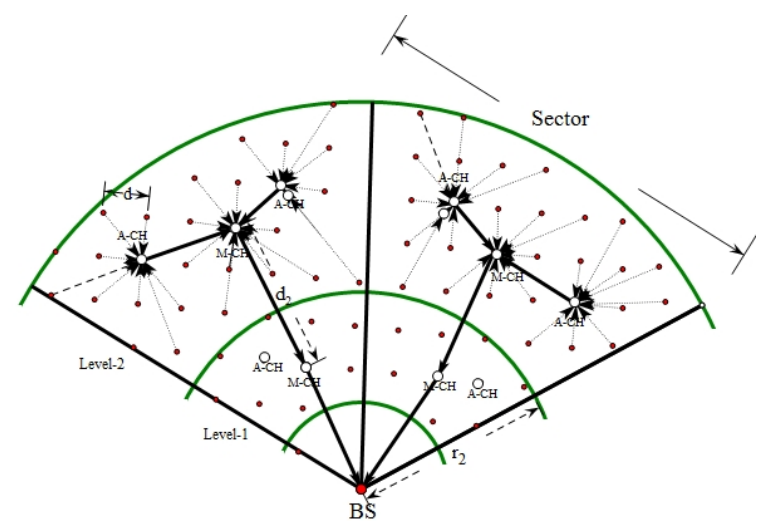

Fig 3. Data Transmitting in MH-TSC

C. Data Transmission. In this phase, the member nodes gather and send data to one of its nearest aux-CHs. The aux-CH fuses the data and transmits to the one-hop aux- $\mathrm{CH}$ in the chain until the data reach to the major- $\mathrm{CH}$. The major- $\mathrm{CH}$ aggregates the data again and transmits to the major- $\mathrm{CH}$ of another cluster in lower level track. Thus, the data are transmitted in multi-hop fashion and finally arrive the BS. For example, the major-CH in level-3 transmit the data to the major-CH in level-2 and then to level-1 and finally to the BS, as shown in Fig.3.

Similar to LEACH [2], the BS breaks the process into lots of rounds. The BS maintains a timer, when a round is nearly ended, the BS broadcasts a message requesting all the nodes report their residual energy and selects the $\mathrm{CHs}$ for the clusters as described in (B) step. When current round is ended, the BS broadcasts the results to all the nodes, the CHs construct the chain again. In this way, the execution of the complex algorithm is done by BS, further reducing the energy consumption, distributing the energy consumption on all the nodes, and prolonging the network life.

\section{Energy Analysis And Performance Evaluation}

A. Energy Analysis of MH-TSC and TSC scheme.The total energy for a transmitter to send a $\mathrm{k}$-bit message over a distance $\mathrm{d}$ is given by Eq.1:

$$
E_{\text {total }}(k, d)=E_{\text {elec }} k+\varepsilon_{\text {amp }} k d^{2}
$$

where $\varepsilon_{\text {amp }}$ is the energy constant for the radio transmission, and $E_{\text {elec }} k$ is the total energy used to run the circuitry to handle k-bit message[9], its value remains constant for a network. $\varepsilon_{\text {amp }} \mathrm{d}^{2}$ is the energy for transmitter to send k-bits over distance $d$, We denote it by the expression $E_{t x}(k, d), d$ is variable, and it is the reason for variable energy consumption among different routing algorithms.

Navin Gautam [7] had analyzed the Energy Relations of PEGASIS, CCS, and TSC, and proved the TSC scheme is more energy efficient as compared to PEGASIS and CCS. We mainly analyze the energy relations of TSC and MH-TSC scheme. 
For TSC scheme, the radio transmission energy consumed to transmit the k-bit data from the source node to the BS is given by Eq.2 [7]:

$$
E_{t x}(k, d)=\varepsilon_{a m p}\left[N_{s}\left(n^{\prime \prime}-1\right) d^{2}+\sum_{i=1}^{N_{s}-1} d_{2}^{2}+r_{2}^{2}\right]
$$

where $r_{2}$ is the distance between the head node and the BS, $N_{s}$ is the number of sectors, $n^{\prime \prime}$ is the average number of nodes in a sector, and $d_{2}$ is the distance between head nodes in two neighboring clusters in the same sector.

Compare to TSC, the MH-TSC scheme shorts the chain, and the member node transmits data to one-hop aux- $\mathrm{CH}$, so the radio transmission energy consumed to transmit the k-bit data from the source node to the BS is displayed as Eq.3:

$$
E_{T x}(k, d)=\varepsilon_{a m p k}\left[N_{s}\left(N_{a}+N_{T}+1\right) d^{2}+\sum_{i=1}^{N_{s}-1} d_{2}^{2}+r_{2}^{2}\right]
$$

where $r_{2}, N_{s}$ and $d_{2}$ is same as TSC, $N_{a}$ is the number of aux-CH in a cluster, and $N_{T}$ is the number of tracks.

Assuming the cluster division of the MH-TSC is similar with TSC, then the difference of their energy consumption are mainly in the path of a node transmitting data. In TSC, all the nodes are in a chain, the data is forwarded and transmitted by many nodes in the chain, but in MH-TSC, the node transmits data to its aux- $\mathrm{CH}$ and aux- $\mathrm{CH}$ forwards the data along the chain, because the number of aux- $\mathrm{CH}$ is small, in other words, $\mathrm{n}^{\prime \prime}>>\mathrm{N}_{\mathrm{a}}+\mathrm{N}_{\mathrm{T}}$, therefore, the path is much shorter than TSC, resulting the MH-TSC scheme is more energy efficient as compared to TSC.

B. Delay Analysis of MH-TSC and TSC scheme. We define the delay as the time consumed by transmitting data from one source node to the BS, mainly including the intra-cluster delay and inter-cluster delay. For MH-TSC and TSC scheme, the inter-cluster is nearly same, so we mainly discuss the intra-cluster delay. For TSC, the process of a node transmitting data in the cluster can be divide into 3 phrases, the node transmits data to its neighbor in the chain, the neighbor fuses the data and then forward to the next neighbor, and so on, until reach the $\mathrm{CH}$. Assuming the message length is $M$ bit, the bandwidth is $B \mathrm{~b} / \mathrm{s}$, the time of fusing data ist ${ }_{0}$, and there are $n$ intermediate nodes from the source node to $\mathrm{CH}$. Because the propagation delay is too small compared with transport delay, so we ignore it. Eq.4 gives the intra-cluster delay of TSC:

$$
\mathrm{T}_{\mathrm{TSC}}=\left(\mathrm{M} / \mathrm{B}+\mathrm{t}_{0}\right)(\mathrm{n}+1)
$$

For MH-TSC, the source node transmits data to its aux-CH, aux-CH fuses the data from the node within its jurisdiction, then transmits to its neighbored aux- $\mathrm{CH}$, the neighbor then forwards to the next aux- $\mathrm{CH}$, until reach the major- $\mathrm{CH}$. assuming one aux- $\mathrm{CH}$ serves s member nodes, and there are $\mathrm{n}^{\prime}$ aux- $\mathrm{CH}$ in the cluster. The intra-cluster delay of MH-TSC is given by Eq.5:

$$
T_{M H-T S C}=\left(M / B+t_{0}\right)\left(n^{\prime}+1\right)
$$

In general, $n>n^{\prime}$, so the TSC has larger intra-cluster delay than MH-TSC.

C. Performance Evaluation. We evaluate the performance of the MH-TSC scheme based on the Matlab platform. For simplicity, we deploy 100 nodes in a sector with BS at center $(0,0)$, set the initial 
energy of each node to $24 \mathrm{~J}$, the number of tracks was set to 5 , the width of the arc area is 50 , the node number in each arc-area are 33,27, 19, 15, and 6 respectively, and the distance between two adjacent nodes in a cluster is 1 . We assume that no energy was consumed when the node stayed idle or went to sleep and the energy was spent only during data transmission and reception. We run the experiments 1000 rounds and ten times data transmitting in each round. The other parameters for the simulation such as transmitter amplifier and data bit are expressed in Table 1, the node number in each cluster is small, so we define the $\mathrm{N}_{\mathrm{a}}$ as 2, but in level-1 cluster, there is only a major-CH responding for forwarding the data from the level 2 and no aux-CHs, and the member nodes transmit data to the BS one-hop.

Table 1. Variables used for simulation

\begin{tabular}{|c|c|}
\hline Parameter & Value \\
\hline$\varepsilon_{\text {amp }}$ & $10^{-12} \mathrm{~J}$ \\
\hline $\mathrm{k}$ & 2000 \\
\hline $\mathrm{N}_{\mathrm{T}}$ & 5 \\
\hline $\mathrm{N}_{\mathrm{s}}$ & 6 \\
\hline $\mathrm{n}^{\prime \prime}$ & 100 \\
\hline $\mathrm{N}_{\mathrm{a}}$ & 2 \\
\hline
\end{tabular}

We compare the proposed MH-TSC scheme with TSC, Fig.4 shows the simulation results for energy consumption over simulation round. The simulation result shows that the MH-TSC scheme consumes lesser energy than TSC scheme and more efficient in conserving the energy. Similarly, Fig. 5 shows that the MH-TSC scheme performs well in terms of number of nodes alive over simulation time. The number of alive nodes after the completion of simulation determines the life of the network. More the number of alive nodes after simulation time, longer will be the life of the network. Also, Fig. 5 shows that the time for the death of the first node is more in case of MH-TSC than the time for the death of the first node in TSC. Therefore, from the simulation results shown in Fig. 5, we can conclude that the network lifetime for proposed MH-TSC scheme is also greater as compared to the network lifetime of TSC.

\section{Conclusions}

In the paper, we propose a MH-TSC scheme to improve the performance of TSC [7]. The evaluation show that the MH-TSC scheme further reduces the energy depletion, prolongs the network and shortens the delay.

\section{Acknowledgments}

This material is based upon work supported by the Inner Mongolia Autonomous Region Natural Science Foundation (Grant No. 2012MS0930), and Inner Mongolia Autonomous Region scientific research projects of colleges and universities (Grant No. NJZY12032). 


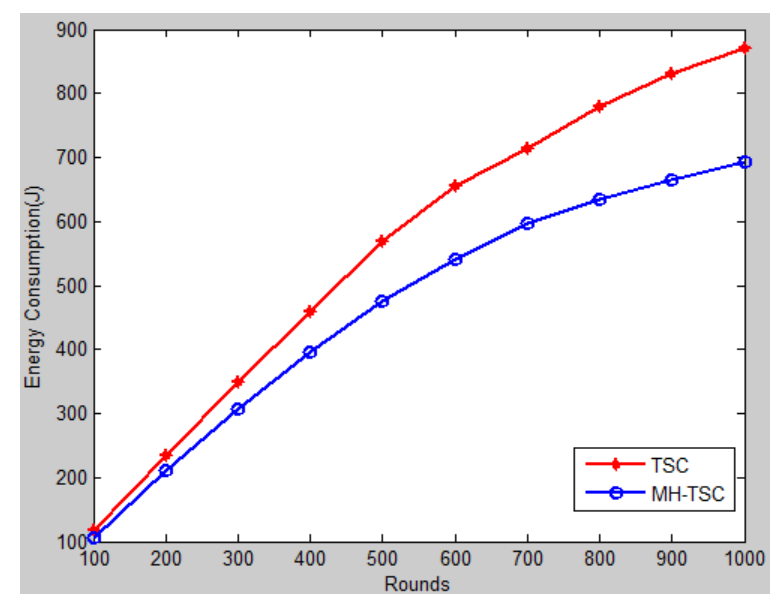

Fig 4. Energy Consumption over rounds

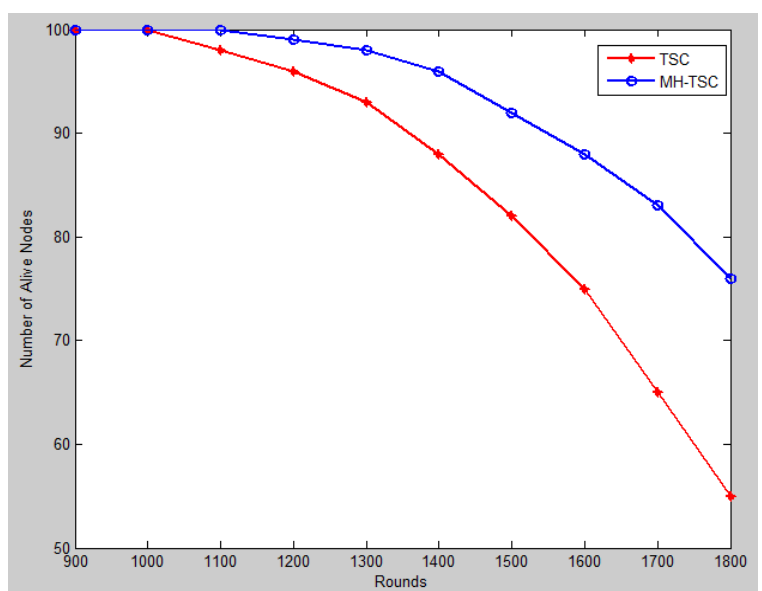

Fig 5. Number of Nodes Alive over rounds

\section{References}

[1] Al-Karaki, J.N. and Kamal, A.E, Routing techniques in wireless sensor networks: A survey, IEEE Wireless. Communication. (11)2004, 6-28.

[2] W. Heinzelman, A. Chandrakasan, and H. Balakrishnan, Energy fficient Communication Protocol for Wireless Micro sensor Networks, in proc. of the 33rd Hawaii International Conf. on System Sciences, (2)2000,10.

[3] W. Heinzelman, A. Chandrakasan, and H. Balakrishnan, An Application-Specific Protocol Architecture for Wireless Microsensor Networks, IEEE Transactions on Wireless Communications, (1)2002,660-670.

[4] F. Xiangning and S. Yulin, Improvement on LEACH Protocol of Wireless Sensor Network, in proc. of 2007 International Conf. on Sensor Technologies and Applications,2007,260-264.

[5] S. Lindsey and C. Raghavendra, PEGASIS: Power-Efficient Gathering in Sensor Information Systems, in proc. of the IEEE Aerospace Conf., (3)2002, 1125-1130.

[6] S.M. Jung, Y.J Han, and T. M Chung, The Concentric Clustering Scheme for Efficient Energy Consumption in the PEGASIS, in proc. of the 9th International Conf. on Advanced Communication Technology, (1)2007, 260-265.

[7] Gautam, N., Lee, W.I. and Pyun, J.Y, Track-Sector Clustering for Energy Efficient Routing in Wireless Sensor Networks, In Proc. of the 9th IEEE International Conference on Computer and Information Technology, 2009, 116-121.

[8] Liang, J., Wang, J. and Chen, J, A Delay-Constrained and Maximum Lifetime Data Gathering Algorithm for Wireless Sensor Networks, In Proc. of the Fifth International Conference on Mobile Ad-hoc and Sensor Networks, 2009,148-155.

[9] D. Song, Probabilistic Modeling of Leach Protocol and Computing Sensor Energy Consumption Rate in Sensor Networks, Technical Report, TR 2005-2-2, 2005. 